\title{
Aspectos epidemiológicos de la diabetes mellitus y su relación con los contaminantes ambientales
}

\author{
Epidemiological aspects of diabetes \\ mellitus and its relationship with \\ enviromental pollutants
}

\begin{abstract}
Biochemical processes involving insulin and its receptor are responsible for regulating carbohydrate and fat metabolism. Disruption of these signaling pathways could lead to obesity, insulin resistance and diabetes, among other health problems. Diabetes has a global impact and its high prevalence has been associated with genetic and environmental factors. Recently, there has been a strong interest in establishing the relationship between type II diabetes and exposure to environmental contaminants, particularly persistent organic pollutants (POPS). This association has is based on epidemiological evidence and results from in vitro and in vivo experiments. In this review, the authors present some of the epidemiological aspects of diabetes, the biochemical pathways involved in insulin action, and how these environmental toxicants have impacted on population, through diverse mechanisms, to produce this disease. While several authors consider that the etiology of diabetes has a very important component derived from exposure to environmental pollutants, it is clear that there is still a substantial amount of work to develop in order to identify cause-effect relationships and the mechanisms involved.

Key words: Organic pollutants; intracellular signaling; insulin; insulin receptor.
\end{abstract}

\section{INTRODUCCIÓN}

La diabetes, es una enfermedad caracterizada por hiperglucemia, dislipidemia y resistencia a la insulina (1-4). Progresa a complicaciones macrovasculares y microvasculares, con deterioro de la calidad y esperanza de vida (5-8). Involucra interacciones entre factores genéticos, culturales y ambientales que pueden generar cambios transcripcionales alterando la secreción y la acción de la insulina, así como el desarrollo y función de las células $\beta$, con consecuencias sobre el metabolismo y la obesidad $(9,10)$.

Existen varios tipos de diabetes, clasificados por su inicio, dependencia a la insulina y otras características. Las más estudiadas son la tipo 1 y 2, sin embargo, la diabetes mellitus tipo 2 (DMT2) representa el 90\% de los casos diagnosticados (11). La tipo 1 es de origen genético, autoinmune, con destrucción de las células $\beta$ del páncreas, y posterior ausencia de producción de insulina (12-15). La tipo 2 ocurre por disfunción de las células $\beta$, resistencia y deficiencia en la producción de insulina
Barbara Arroyo-Salgado, Jesus Olivero-Verbel

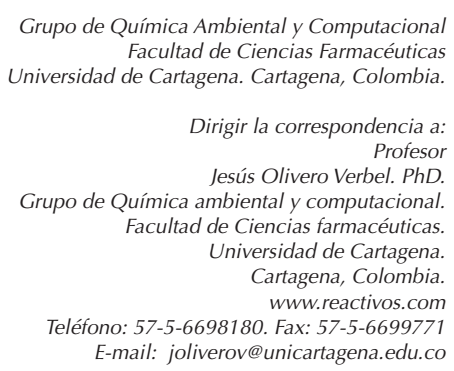

Este trabajo fue recibido el 24 de Septiembre de 2013 y aceptado para ser publicado el 18 de Enero de 2014.

(15), con frecuencia influenciada por factores que involucran desequilibrio en la ingesta, gasto energético y estilo de vida (16), convirtiéndola en pandémica (17).

Este artículo es el resultado de una revisión temática, en su gran mayoría de literatura publicada entre 1955 y 2012, sobre la relación entre la epidemiología de la DMT2 y la contaminación ambiental, haciendo énfasis en los mecanismos de toxicidad involucrados. Para la minería de textos se utilizaron varias bases de datos, incluyendo Pubmed, Medline, Lilacs y Scielo, realizando la búsqueda empleando las palabras claves "diabetes", "COPs", "contaminación ambiental" y "DMT2".

1. Epidemiología de la diabetes mellitus. La diabetes mellitus es devastadora para países con economías débiles $(18,19)$, hecho que ha despertado el interés de la Organización Mundial de Salud (WHO), la Asociación Americana de Diabetes (ADA), y la Federación Internacional de Diabetes (IFD), entre otras instituciones. Afecta 366 millones personas 
en el mundo y está previsto que 552 millones en 2030 la padezcan en Asia, India, América Latina, Oriente Medio y el África Sub-Sahariana $(20,21)$.

El país con la más alta prevalencia es Micronesia (Isla de Nauru), donde el $31 \%$ de sus habitantes tiene diabetes, aunque países tales como Emiratos árabes unidos, Bahréin, Kuwait, Omán y Egipto poseen una prevalencia promedio del $27 \%$ (22). La prevalencia en los EE.UU es $8.0 \%$ y ha sido estimado que para Reino Unido y Australia la enfermedad crece en paralelo a la obesidad (23). Así mismo, en África, Oriente medio y central, así como en Sur América, la prevalencia se incrementará en cerca del 80\% en los próximos 15 años (24).

Desde el punto de vista económico, el tratamiento de la DMT2 y de sus complicaciones alrededor del mundo costó alrededor de 232 billones de dólares para el año 2007, y para el año 2025 esté valor ascenderá a más de 302. De manera similar, el mercado global del tratamiento de la DMT2 fue de 26.3 billones de dólares para el año 2009 y se espera que aumente a más de 34.5 para el $2013(25,26)$. Según la ADA, el costo de la diabetes en los EEUU es 245 billones de dólares, lo cual incluye 176 en costos médicos directos y 69 en pérdida de productividad (27). Por su elevada morbilidad y mortalidad, cada año suceden 3.8 millones de fallecimientos, lo que equivale a una muerte cada 10 segundos (28), ocurriendo el $80 \%$ en países de bajos y medianos ingresos (29).

La DMT2, por sus proporciones epidémicas en todo el mundo, es considerada una de las peores amenazas mundiales para la humanidad, lo cual probablemente se debe a su naturaleza multietiológica. Los factores de riesgo más destacados son los étnicos (30-32), genéticos (33), ambientales (13), exposiciones ocupacionales y contaminantes ambientales (34-38). Aunque no es posible desconocer su naturaleza plurifactorial, la diabetes parece estar asociada con el estilo de vida, el bajo nivel socioeconómico, la edad, la obesidad, la falta de actividad física, la carga glucémica en la dieta, el estrés, la condición sedentaria, y una dieta poco saludable (39), entre otros.

En este escenario, la intervención de los factores ambientales ha puesto de manifiesto opiniones diversas al respecto de la etiología y patogénesis de la DMT2, estableciendo vínculos con las rutas biológicas celulares e implicando de manera directa a la insulina y a su receptor, como moléculas determinantes en las alteraciones de transducción de señales conducentes a la enfermedad. A continuación aparece una descripción del sistema de señalización de la insulina.

2.0. Sistema de transducción de señales activado por la insulina. La insulina es una proteína de 51 aminoácidos (5808 Da) que forma un hexámero. Posee dos cadenas $\alpha$ y $\beta$, tres $\alpha$-hélices, dos en la cadena $\alpha$ y uno en la cadena $\beta$, y un centro hidrofóbico, así como aminoácidos no polares, que son importantes para el plegado y el mantenimiento de la estructura (40). Ambas cadenas están unidas por enlaces disulfuro entre los residuos $\alpha 7-\beta 7$ y $\alpha 20-\beta 19$. Además, la cadena $\alpha$, posee otro puente disulfuro en la posición $\alpha 6-\beta 11$. En presencia de zinc, los monómeros se ensamblan para formar hexámeros, donde cada uno de los dos iones centrales de zinc está coordinado por tres residuos de His $\beta 10$ (41).

Después de la ingesta de una comida, suceden tanto un incremento de glucosa como del metabolismo. La glucosa es movilizada por los transportadores de glucosa (GLUT4) al citosol donde se inicia la glucólisis $(42,43)$, pasando por el ciclo de Krebs, y terminando en la generación de trifosfato de adenosina (ATP), ocasionando la inhibición de canales de potasio sensibles al ATP (canal K-ATP). El resultado es la depolarización de la membrana celular, seguido de la apertura de los canales de $\mathrm{Ca}^{2+}$ dependientes de voltaje. La concentración de $\mathrm{Ca}^{2+}$ citoplasmático libre y elevado, finalmente desencadena la secreción de insulina por las células- $\beta$ (islotes de Langerhans del páncreas). La insulina secretada al interaccionar con su receptor celular suprime la gluconeogénesis hepática, promueve la síntesis de glicógeno y el almacenamiento de este último en el hígado, músculo y tejido adiposo $(6,44)$. Esta hormona igualmente interviene en múltiples actividades biológicas tales como el control de la homeostasis de glucosa, vías de señalización intracelular (45), metabolismo de lípidos, síntesis de proteínas, control de enzimas-hormonas $(15,44,46)$ y la expresión de múltiples genes $(47,48)$, entre otros procesos.

2.1. El receptor de insulina ( $R I, \alpha 2 \beta 2)$. Es una proteína de membrana que al activarse por el contacto con insulina activa un sistema de transducción de señales cuya alteración puede conducir a patologías como la DMT2, arterioesclerosis, enfermedad coronaria, vascular, dislipidemia, infertilidad femenina y obesidad, entre diversos problemas (49). Aparece en dos isoformas: la A es de expresión prenatal, tiene afinidad por el factor de crecimiento de tipo insulina (IGF)-II y contribuye a la proliferación celular. La isoforma $B$, se expresa en el tejido diferenciado adulto y posee afinidad por la insulina, siendo responsable de los efectos metabólicos de esta hormona (50).

A pesar que los tejidos diana principales de la insulina son el hígado, el tejido adiposo y el músculo esquelético, los RI también se han encontrado en el cerebro, corazón, riñón, alvéolos pulmonares, acinos pancreáticos, endotelio vascular, placenta, monocitos, granulocitos, eritrocitos, y fibroblastos (50). La presencia reducida de RI en el hígado y su hiperfosforilación en serina /treonina, distinguen el desarrollo de resistencia a la insulina y DMT2 (51).

El RI conserva 4 dominios, uno de anclaje a la membrana, una fosfotirosina vinculante, uno con actividad tirosina fosfatasa 2 y otro regulador de unión a cinasa (52). Además posee un promotor y elementos de respuesta a distintos factores transcripcionales $(53,54)$, con múltiples sitios de iniciación transcripcional, útiles para el factor transcripcional SP1 (factor de transcripción humano implicado en la expresión génica).

El gen del receptor de la insulina está controlado por varios factores de transcripción, tales como Ch/REBP, NF, IRNF-I (por sus siglas en inglés: "Carbohidrate response element binding protein", nuclear factor, e "insulin receptor nuclear factor-I", respectivamente). Esta modulación responde a glucocorticoides (GREs), mineralocorticoides, adenosín mono fosfato cíclico (AMPc), factor transcripcional del RI específico de hepatocitos (HTFIR), factor transcripcional específico del hígado (LF-A1) y un elemento regulador negativo (55-58), entre otros.

2.2. Activación del receptor de insulina (RI). Tras la unión de la insulina al RI, éste sufre un cambio conformacional e inicia la transducción de señales y su regulación. El proceso empieza con un cambio en la fosforilación en los residuos citoplasmáticos de la tirosina de algunas proteínas del citosol, o del núcleo, incluyendo a sus substratos denominados IRS-1 hasta IRS6 $(59,60)$. Estos sustratos, difieren entre sí por su distribución, localización subcelular, desarrollo de la expresión, unión al receptor de insulina e interacción con proteínas que contienen un dominio $\mathrm{SH} 2$, desempeñando funciones diferentes y específicas in vivo (55).

IRS-1 y IRS-2 son integrados a la señal de la insulina y junto al receptor del factor de crecimiento tipo 1 similar a 
la insulina (IGF-IR), que es una tirosina-cinasa del receptor, juegan un papel fundamental en la señalización de la supervivencia y proliferación celular $(49,61)$. Activan fosfoinosítidos3-cinasa (PI3K) y promueven la proliferación celular a través de la activación de la cascada de la proteína cinasa activada por mitógenos (MAPK), bloqueando la apoptosis mediante la inducción de la fosforilación y la inhibición de las proteínas pro-apoptóticas. Inmediatamente después de su fosforilación, estas proteínas interactúan con moléculas de señalización intracitosólicas, dando lugar a una amplia serie de vías de señalización molecular en el citosol, las cuales transmiten el mensaje de la insulina hacia factores de transcripción en la membrana nuclear (52).

Esta activación molecular incluye dos vías principales: la fosfatidilinositol-3-OH cinasa estimulada por insulina (PI-3K) y la vía de la proteína cinasa activadora de la mitogénesis (MAP) $(52,55,61-63)$. Esas proteínas actúan como reguladoras vitales camino abajo "downstream". Su acción, en conjunto con la activación de la fosfoinositol-3 cinasa ( $\mathrm{PI}-3 \mathrm{~K})$ /proteína cinasa $B$, o PKB (Akt), señalan la vía y la translocación subsecuente de vesículas de almacenamiento intracelular (GLUT4) hasta la membrana plasmática.

2.3 Vía metabólica o vía de la fosfatidilinositol-3-OH cinasa estimulada por insulina (PI-3K). En los mamíferos esta vía facilita la captación de glucosa dependiente de insulina en el tejido adiposo y músculo, y el interés en la misma radica en que está implicada en enfermedades humanas tales como la diabetes, convirtiéndose en un objetivo para la intervención terapéutica $(63,64)$. En tejidos como el músculo esquelético e hígado, el RI sufre autofosforilación (65) y conlleva a la fosforilación de los IRS $(62,66)$. Estas proteínas fosforiladas intervienen en las cascadas corriente abajo (55), donde son reclutadas por la fosfatidilinositol- 3-cinasa (PI3K) y unidas a un sitio de acoplamiento de la serina/treonina cinasa Akt, para promover el tráfico del transportador de glucosa GLUT4 hacia la superficie de la célula $(67,68)$.

Cabe destacar que la mayoría de los efectos metabólicos de la insulina están regulados por Akt. Por ejemplo, la fosforilación de Akt-dependiente de cinasa glicógeno sintasa 3 (GSK-3ß), conduce a la activación de la glicógeno sintasa (GS), con lo cual se mejora el almacenamiento de la glucosa en forma de glucógeno. Akt posee tres isoformas en los mamíferos; cada una comparte el mismo dominio, y su organización está codificada por genes separados, con un $85 \%$ de homología entre ellas (69). La expresión de Akt-1 es por doquier, mientras que Akt2 es mayor en los tejidos que responden a la insulina, tales como corazón, hígado, riñón y músculo esquelético (70). Por su parte, Akt3 aparece limitada al cerebro y los testículos.

El variado perfil de expresión de las diferentes isoformas de Akt ha planteado la posibilidad de papeles singulares en los tejidos específicos de señalización. Akt además participa en la integración de las respuestas anabólicas y catabólicas de transducción de las señales que emanan de los factores de crecimiento, los nutrientes, las citoquinas, y la contracción muscular, a través de cambios en la fosforilación de sus numerosos sustratos (71). Dada su multifunción, Akt-1 podría jugar un papel fundamental en actividades tales como el envejecimiento, trastornos en el metabolismo celular y la homeostasis fisiológica (72).

2.4 Vía mitogénica o vía de la proteína cinasa activadora de la mitogénesis (MAP). Las proteínas cinasas activadas por mitógenos (MAPKs) regulan diversos programas celulares por transmisión de señales, convirtiendo los estímulos extracelulares en una amplia variedad de respuestas funcionales.
Las células eucariotas poseen múltiples vías MAPK, que coordinadamente regulan la expresión génica, la mitosis, el metabolismo, la motilidad, la supervivencia, la apoptosis y la diferenciación (73).

Esta vía es activada por la unión de la proteína de unión al factor de crecimiento 2 (Grb2), o al IRS vía su dominio $\mathrm{SH} 2$. A su vez, Grb2 se encuentra ligada a una proteína de intercambio de nucleótidos denominada "Son of Sevenless" o mSOS de mamíferos, que cataliza el intercambio de GDP por GTP en una pequeña proteína GTPasa denominada Ras (p21), resultando en su activación. La proteína Ras activada es unida a la parte interna de la membrana plasmática atrayendo la región NH2-terminal de otra proteína conocida como Raf. La interacción Ras-Raf permite la fosforilación de Raf por un número de cinasas Ser/Thr, desinhibiendo y liberando de esta manera la cinasa Raf (74). Raf activa a las tirosina/treonina cinasas duales denominadas proteína cinasa activada de mitógenos 1 (MEK1). Éstas finalmente fosforilan a las serín treonín cinasas 1 y 2 (ERK1/2). Este paso es sumamente importante, ya que ERK, puede fosforilar diferentes proteínas dianas localizadas en la membrana plasmática y en el citoplasma, dando lugar a la activación de otras vías de señalización o pasar al núcleo, para allí, regular la transcripción, modificando la actividad de proteínas tales como c-Myc, c-Jun, c-Fos, entre otros, y modulando así la expresión de distintos genes (75).

En términos generales, estas vías moleculares actúan de forma integrada y con alta precisión para coordinar la regulación del tráfico vesicular, la síntesis de proteínas, la activación e inactivación de enzimas clave y principalmente la expresión genética, cuyo resultado final es la regulación del metabolismo de la glucosa, lípidos y proteínas. Sin embargo, estas vías pueden también ser intervenidas de manera negativa, ocasionando que en la actualidad exista una preocupación a nivel mundial alrededor de la forma cómo los productos químicos presentes en el medio ambiente están contribuyendo al aumento global de las enfermedades cardiometabólicas crónicas como la DMT2 (76), logrando convertirse en una epidemia incontrolada asociada a elevada morbilidad y mortalidad (http://www.idf. org/fact-sheets/diabetes-cvd). A continuación una descripción de esta asociación es discutida en mayor detalle.

3.0. DMT2 y la contaminación ambiental. Existen aproximadamente once millones de compuestos químicos disponibles, pero sólo 3000 son producidos comercialmente, por lo que suelen encontrarse en efluentes residenciales, industriales y urbanos, representando fuentes de contaminación ambiental (77). Reconocidos por el Programa de Medio Ambiente de las Naciones Unidas en el Convenio de 1995 de Estocolmo, en su mayoría son denominados compuestos orgánicos persistentes (COPs) ó "POPs" (Persistent Organic Pollutants). En este grupo se destacan las dioxinas, los furanos, los bifenilos policlorados (PCB) y algunos pesticidas, los cuales por sus efectos adversos fueron prohibidos en $1970(3,78,79)$. Estos compuestos poseen características tales como lipofilicidad, persistencia, baja degradación y toxicidad (80), y están asociados a teratogenecidad, disrupción endocrina, bioacumulación en el tejido adiposo, resistencia a la insulina y diabetes (81). Algunos datos generales de estas sustancias, considerados COPs de acuerdo con la Convención de Estocolmo 2001, 2009 y 2011, son presentados en la tabla 1 (82). Otros compuestos que han ganado un lugar como factores etiológicos de la diabetes son los plaguicidas organofosforados y los disruptores endocrinos derivados de plásticos, en particular los ftalatos y el bisfenol A (BPA), entre otros. 
TABLA 1

Contaminantes orgánicos persistentes de acuerdo con la Convención de Estocolmo 2001, 2009, 2011.

Químicos

Tipo

PubChem ID*

Los contaminantes orgánicos persistentes (COPs), 2001, 2009 y 2011

1

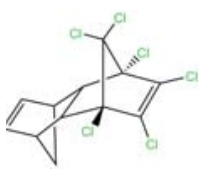

Aldrin

CAS:309-00-2

2

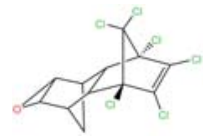

Dieldrin

CAS:60-57-1

3

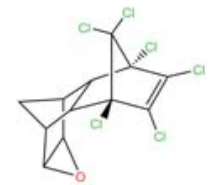

Endrin

CAS:72-20-8

4

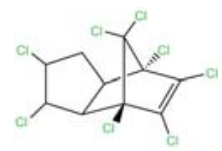

Clordano

CAS:57-74-9

5

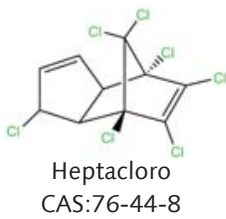

6

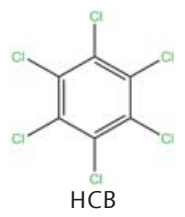

CAS:118-74-1

7

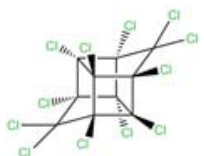

Mirex

CAS:2385-85-5
Pesticida

12310947

Pesticida

969491

Pesticida

46174049

Pesticida

5993

Pesticida

3589

Pesticida y de uso industrial

8370

Pesticida

16945 
8

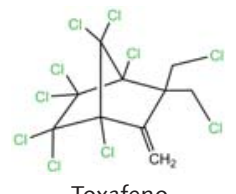

Toxafeno

CAS:8001-35-2

9

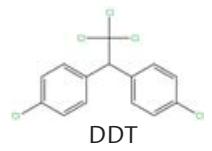

CAS:50-29-3

10<smiles>Clc1cccc(-c2ccccc2)c1</smiles>

CAS:2051-60-7<smiles>Cc1cc2c(cc1I)Oc1cc(Cl)c(Cl)cc1O2</smiles>

TCDD

CAS:1746-01-6

11 y 12

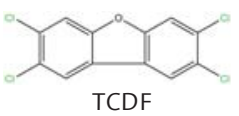

CAS:1746-01-6

13

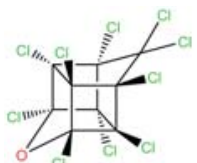

Clordecona

CAS:143-50-0

14

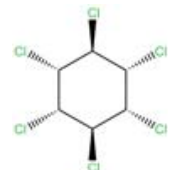

Lindano $(\mathrm{Y}-\mathrm{HCH})$

CAS:58-89-9

15

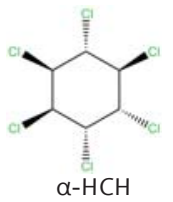

CAS:319-84-6

16

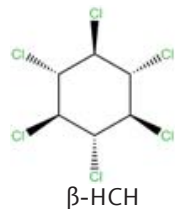

CAS:319-85-7

\section{Pesticida}

5284469

Pesticida

3036

Productos químicos industriales y subproductos

249266

15625

Subproductos

Pesticida

299

Pesticida

727

Pesticida y subproductos

727

Pesticida y subproductos

727 
17

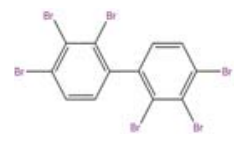

Hexabromobifenilo

CAS: 82865-89-2

18

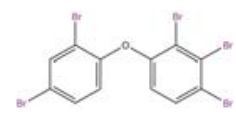

Penta-BDE

CAS: $182346-21-0$

19<smiles>Cc1cc(Oc2cc(C)c(C)c(C)c2C)c(C)c(C)c1C</smiles>

Hepta-BDE

CAS:207122-16-5

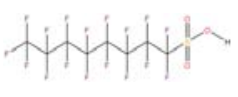

PFOS

20

CAS:1763-23-1

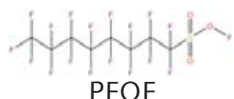

307-35-7

21<smiles>Clc1cc(Cl)c(Cl)c(Cl)c1Cl</smiles>

Pentaclorobenceno

CAS:608-93-5

22

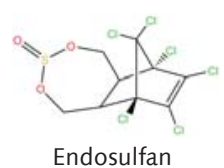

Endosulfan

CAS:115-29-7
Productos químicos industriales

154482

Productos químicos industriales

177368

Productos químicos industriales

15509899

Productos químicos industriales

74483

9388

Pesticida, productos químicos industriales y subproductos

11855

Pesticida

3224
3.1. COPs y otros compuestos relacionados con DMT2. Los COPs han sido reportados en humanos con diabetes (83), para los cuales aparecen concentraciones séricas elevadas (8387). Así mismo, en animales se ha puesto de manifiesto su presencia y asociación con obesidad abdominal, resistencia a la insulina, y hepatoesteatosis, confirmado con estudios en adipocitos cultivados en presencia de plaguicidas organoclorados, y evidencia derivada de la inhibición de la acción de la insulina (88) y reducción de la captación de la glucosa (89).

Numerosos reportes siguen aportando bases a estas relaciones: Los de carácter transversal con asociaciones significativas $(83,84,90)$; los prospectivos sugieren que la exposición a los COPs podría aumentar el riesgo de DMT2 $(90,91)$. Sin embargo, todavía existe escepticismo y controversia en ellos $(90,92)$. En algunos casos, las inconsistencias de los estudios se han atribuido a la poca cantidad de muestras, diferentes orígenes de exposición, distribución y otras características de las poblaciones estudiadas (72). Algunos de los COPs usualmente implicados en DMT2 aparecen descritos a continuación.

3.1.1. Dioxinas: Bifenilos policlorados (PCBs), dibenzodioxinas y dibenzofuranos policlorados (PCDDs/PCDFs). Los PCBs son sintéticos y un grupo de ellos, los denominados no-orto-PCBs, tienen propiedades similares a las dioxinas. Por su parte, las dioxinas y furanos usualmente provienen de procesos térmicos e industriales (incineración de residuos, combustión inadecuada, producción de químicos (refrigerantes, lubricantes), herbicidas clorados orgánicos y blanqueo de papel (OMS, IRAC). Estos compuestos se distribuyen en la atmósfera y en el suelo; ingresan por ingestión (leche y sus derivados, pescado y agua) (91) e inhalación, ejerciendo su 
toxicidad a través de la activación del receptor de hidrocarburos aromáticos (AhR) (93). Intervienen en rutas biológicas determinantes en procesos de carcinogénesis, reproducción, toxicidad hepática, enfermedades tales como aterosclerosis, hipertensión, hiperlipidemia y diabetes (3).

Investigaciones tanto in vitro como in vivo, implican a las dioxinas en la disminución en la captación de glucosa, la producción de insulina y en la prevalencia de la diabetes (89). Existe evidencia de niveles altos de dioxinas en suero de trabajadores con diabetes (12), y personas que viven en lugares residenciales junto a residuos de COPs (94). Además, la diabetes se ha relacionado con la post-exposición a dioxinas en diferentes lugares tales como en Seveso Italia (95); Corea (31); Bélgica; Michigan (37) y Japón (87). Otros estudios han señalado que la exposición a bajos niveles de dioxinas aumenta el riesgo de diabetes, hipertensión e hiperlipidemia (96-98).

3.1.2. Los policlorobifenilos (PCB) o bifenilos policlorados. Son químicos sintéticos, persistentes, bioacumulables y pueden biomagnificarse en la cadena alimentaria (3). Están presentes en transformadores, condensadores eléctricos, fluidos hidráulicos, aditivos de aceite para pinturas, calafateo de ventanas, techo o pavimento $(3,78)$. Para los PCBs existen 209 congéneres posibles, los cuales han sido clasificados como tipo "dioxinas" y tipo "no dioxinas" (79). Estudios en animales, han mostrado que estas moléculas generan cambios bioquímicos en las células $\beta$ pancreáticas $(35,36)$, así como disminución de la sensibilidad a la insulina y alteraciones en la homeostasis de la glucosa, el metabolismo de lípidos, y en la función tiroidea (99).

Algunos PCBs pueden imitar la acción de la hormona tiroidea y de los estrógenos. Por ejemplo, el PCB 77 ha sido asociado con la diferenciación de adipocitos, promoción de la expresión de citoquinas proinflamatorias, cambios en la expresión del receptor tipo gama para la activación de la proliferación de peroxisomas (PPARY) y obesidad (100). Sin embargo, parece existir una correlación negativa entre el PCB 153 y adiponectina en las mujeres obesas, sugiriendo una posible supresión de la liberación o producción de adiponectina. Estudios en trabajadores expuestos a dioxinas, PCB 126 y p, p'DDT, mostraron asociación significativa con diabetes, aunque sólo PCB 126 y p, p'-DDT se asociaron significativamente con diabetes no diagnosticada (3).

Incidencia alta de diabetes también ha sido observada en mujeres víctimas de Yucheng, conocida como la "enfermedad del aceite", que habían estado expuestas a PCBs y PCDFs, y diagnosticadas con cloracné (101). Reportes para Michigan (USA), han mostrado que pacientes con PCBs en suero, están en riesgo para la diabetes del adulto (37). Estos datos fueron registradas igualmente por Turyk (2009) (102) y Patel (2010) (76), quienes reportaron datos similares. En alimentos pesqueros de Suecia, específicamente en la grasa del pescado del Mar Báltico, fue demostrada la presencia de di-orto congéneres de $\mathrm{PCB}$, tales como PCB-153, el cual tendría un efecto diabetógeno por sí mismo (84). Por último, Lee (2012) (92) ha sugerido que los COPs acumulados en el tejido adiposo, en lugar de la obesidad en sí, puede estar críticamente implicados en la patogénesis de la DMT2.

3.1.3. Pesticidas organoclorados. En este grupo se destacan algunos tóxicos como el diclorodifeniltricloroetano (DDT), su metabolito, el diclorodifenildicloroetileno (DDE) y el $\beta$-hexaclorociclohexano ( $\beta$-HCH) (94).

EI DDT es un químico sintético, efectivo para el control de vectores y enfermedades agrícolas (103). Su presencia en concentraciones elevadas fue elevada en personas con diabetes
(34) y en poblaciones que residen en el suroeste de los Estados Unidos (104), este último trabajo fue revisado y comprobado posteriormente en NHANES 1999-2002 (81). Otros estudios igualmente han reportado esta asociación (3, 38, 81, 93, $102,103)$; incluso podría ser atribuible al consumo de peces contaminados con pesticidas.

El HCH posee varias isómeros, siendo los más comunes en las formulaciones comerciales $\alpha-\mathrm{HCH}(53-60 \%), \beta-\mathrm{HCH}$ (3-14\%), $ү-\mathrm{HCH}(11-18 \%), \delta-\mathrm{HCH}(6-10 \%)$ у $\varepsilon-\mathrm{HCH}(3-5 \%)$. Estudios en Estados Unidos han mostrado una asociación significativa entre diabetes y los niveles séricos de $\beta-\mathrm{HCH}$ y otros plaguicidas organoclorados (103).

Aunque muchas publicaciones sustentan un papel preponderante de los COPs en la diabetes, otros compuestos no persistentes están Ilamando la atención de los investigadores, los cuales son brevemente presentados a continuación:

3.1.4. Pesticidas organofosforados. Se destaca en este grupo el Paratión, el cual puede inducir un estado prediabético en ratas Sprague-Dawley, asociación que resultó dependiente del sexo (sexo-selectivo) (105).

3.1.5. Bisfenol A (BPA). Este compuesto es considerado un estrógeno ambiental por excelencia, y un componente común en plásticos de policarbonato, resinas epoxi y de aditivos para la polimerización. Es reconocido por su exposición humana generalizada, habiéndose asociado con trastornos metabólicos como la diabetes mellitus $(106,107)$. Las fuentes de exposición pueden incluir latas de comida, biberones y los selladores dentales (108-110). Estudios in vitro y en animales, demuestran que dosis bajas de BPA alteran la señalización del calcio en el páncreas y su función celular, causando resistencia a la insulina (111) e hiperinsulinemia postprandial (112). El BPA también inhibe la síntesis de adiponectina y podría estimular la liberación de adipoquinas inflamatorias como la interleucina-6 (IL-6) y el factor de necrosis tumoral alfa (TNF- $\alpha$ ) desde el tejido adiposo humano, sugiriendo su vínculo con obesidad y el síndrome metabólico (113).

3.1.6. Ftalatos. Estos químicos son parte de la materia prima principal en la fabricación de plástico. Existen reportes que sugieren una relación entre los ftalatos y la diabetes, obesidad y resistencia a la insulina (114). Estudios in vitro, indican que afectan la regulación del RI, la oxidación de la glucosa en células hepáticas y en general la homeostasis de la glucosa (115). En animales de ambos sexos, la exposición prenatal a ftalatos de diisobutilo reduce la leptina y la insulina sérica (116). También se ha demostrado que participan en el incremento de la peroxidación lipídica, stress oxidativo y el deterioro de la inducción de la tolerancia a la glucosa.

4.0 Mecanismos de los COPs/Diabetes. La asociación entre los COPs y la diabetes todavía no está clara. Sin embargo, ha sido explicada a través de diferentes mecanismos, aunque por fuera de una relación clásica causa-efecto. Existen algunas explicaciones biológicas tales como cambios en la expresión génica, alteración del metabolismo de lípidos, esteroides y del transporte de glucosa, cambios en la vía de señalización de la insulina, perturbación del sistema endocrino, antagonismo de receptores PPAR, la inducción de TNF- $\alpha$, entre muchos otros mecanismos propuestos. Un resumen de los mismos aparece expuesto en la tabla 2.

\section{CONCLUSIÓN}

Existe un cúmulo importante de evidencias científicas de tipo bioquímico y epidemiológico que sugieren una asociación entre los contaminantes ambientales y la diabetes. Es necesa- 
rio, por tanto, realizar estudios epidemiológicos prospectivos y experimentos in vitro e in vivo que ofrezcan una visión más amplia y detallada de este problema.

\section{RESUMEN}

Los procesos bioquímicos que involucran la insulina y su receptor son los responsables de regular el metabolismo de carbohidratos y grasas. La alteración de estas vías de señalización puede conllevar a obesidad, resistencia a la insulina y diabetes, entre otros problemas de salud. La diabetes impacta a nivel global y su alta prevalencia ha sido asociada con factores genéticos y ambientales. Recientemente ha surgido un amplio interés por establecer la relación existente entre la diabetes tipo 2 y la exposición a contaminantes ambientales, en especial los compuestos orgánicos persistentes (COPs). Esta asociación está basada en evidencia epidemiológica y resultados de experimentos in vitro e in vivo. En esta revisión los autores presentan algunos de los aspectos epidemiológicos de la diabetes, las rutas bioquímicas que participan en la acción de la insulina, y como estos tóxicos ambientales han impactado sobre la población, a través de diversos mecanismos, para producir esta enfermedad. Si bien algunos autores consideran que la etiología de la diabetes tiene un componente importante derivado de la exposición a contaminantes ambientales, es claro que aún falta mucho por desarrollar para identificar verdaderas relaciones causa-efecto y los mecanismos involucrados.

Palabras clave: Contaminantes orgánicos; señalización intracelular; insulina; receptor de insulina.

\section{BIBLIOGRAFÍA}

1. McCarthy MI. Progress in defining the molecular basis of type 2 diabetes mellitus through susceptibility-gene identification. Human molecular genetics. 2004; 13 Spec

TABLA 2

Algunos mecanismos implicados en la acción de COPs sobre diabetes.

Mecanismos de los COPs / Diabetes

Cambios en la expresión génica

Alteración del metabolismo de los lípidos

Alteración del transporte de glucosa

Alteraciones en la vía de señalización de la insulina

La inducción de factor de necrosis tumoral $\alpha$ (TNF- $\alpha$ )

Alteraciones en la acción de estrógenos

Susceptibilidad genética asociada con IRS-1
Algunos COPs están involucrados en el aumento o la disminución de la expresión de genes participantes en la homeostasis de la glucosa, lípidos y la acción de la insulina. Entre ellos los genes Srebp 1c (elemento regulador proteína esteroles 1c), FAS (sintasa de ácido grasos), Gen 1 inducido por insulina (Insig-1), lipin 1 (88), coactivador de PPARa (Pgc1 $\alpha)(117)$, PPARy, citrato sintasa (CS), acil CoA deshidrogenasa de cadena media (MCAD) y succinato deshidrogenasa (Sdha), estos últimos asociados con alteraciones en la función mitocondrial y capacidad oxidativa hepática (88).

Los COPs aumentan el riesgo de desarrollar resistencia a la insulina y trastornos metabólicos en animales (88) y humanos (116). Sin embargo, no está claro si el aumento de la adiposidad es un efecto directo, como se sugiere en estudios in vitro, o es un efecto indirecto mediado por la alteración metabólica provocada por ciertos COPs $(100,118)$.

La activación del receptor de hidrocarburos aromáticos (AhR) por algunos COPs tipo dioxina genera cambios en la transcripción del transportador de glucosa (GLUT) $(88,119,120)$. El GLUT4 ocupa un papel central en la DMT2 (121). Sin embargo, su ausencia a nivel del tejido adiposo, conlleva a resistencia a la insulina y la diabetes (122). Las dioxinas igualmente generan cambios en la función del factor nuclear kappa $\beta$, los cuales se han relacionado con diabetes y obesidad (120). Bisfenol A (BPA), por parte, afecta el transporte de glucosa in vivo (122).

Las dioxinas alteran la secreción de insulina estimulada por glucosa en los islotes a través de la vía de señalización del $\operatorname{AhR}(123,124)$, mientras que los ftalatos lo hacen a través de los receptores nucleares. De esta manera afectan la diferenciación de adipocitos, el metabolismo lipídico y resistencia a la insulina, aumentando el riesgo de desarrollo de diabetes (125).

TNFa está involucrado en la resistencia a la insulina (126). Los COPs tipo dioxinas estimulan la expresión de TNF-a y lipoproteína lipasa (LPL) (127).

DDT y algunos de sus metabolitos son reconocidos agentes estrogénicos (128), los cuales pueden modular la deposición de lípidos y por tanto son un factor de riesgo en el desarrollo de diabetes.

IRS-1 está vinculado a la acumulación de lípidos en el músculo y a la disminución de insulina estimulada por el transporte de glucosa (129). Polimorfismos del IRS-1 y de los genes transportadores de glucosa están asociados con DMT2 (130). Esta podría ser una explicación parcial de la susceptibilidad genética a la DMT2. 
No 1:R33-41.

2. Sullivan PW, Morrato EH, Ghushchyan V, Wyatt HR, Hill JO. Obesity, inactivity, and the prevalence of diabetes and diabetes-related cardiovascular comorbidities in the U.S., 2000-2002. Diabetes Care. 2005; 28(7):1599-603.

3. Everett CJ, Frithsen I, Player M. Relationship of polychlorinated biphenyls with type 2 diabetes and hypertension. J Environ Monit. 2011; 13(2):241-51.

4. DeFronzo RA. Pathogenesis of type 2 diabetes mellitus. Med Clin North Am. 2004; 88(4):787-835.

5. King H, Aubert RE, Herman WH. Global burden of diabetes, 1995-2025: prevalence, numerical estimates, and projections. Diabetes Care 1998; 21(9):1414-31.

6. Bloomgarden ZT. European Association for the Study of Diabetes Annual Meeting, 1999: treatment modalities. Diabetes Care. 2000; 23(7):1012-7.

7. Wannamethee SG, Shaper AG, Lennon L. Cardiovascular disease incidence and mortality in older men with diabetes and in men with coronary heart disease. Heart. 2004; 90(12):1398-403.

8. Amos AF, McCarty DJ, Zimmet $P$. The rising global burden of diabetes and its complications: estimates and projections to the year 2010. Diabetic Med 1997; 14 (Supp/ 5): S1-85.

9. Tusié Luna MT. Genes and type 2 diabetes mellitus. Arch Med Res. 2005; 36(3):210-22.

10. Hu FB. Globalization of diabetes: the role of diet, lifestyle, and genes. Diabetes Care. 2011; 34(6):1249-57.

11. Franco-Hincapié $L$, Duque CE, Parra MV, Gallego N, Villegas A, Ruiz-Linares A, Bedoya G. Association between polymorphism in uncoupling proteins and type 2 diabetes in a northwestern Colombian population. Biomédica. 2009; 29(1):108-18.

12. Longnecker MP, Daniels JL. Environmental contaminants as etiologic factors for diabetes. Environ Health Perspect. 2001; 109 (Suppl 6):871-6.

13. Chiasson JL, Rabasa-Lhoret R. Prevention of type 2 diabetes: insulin resistance and beta-cell function. Diabetes. 2004; 53 Suppl 3:S34-8.

14. Knip $M$, Veijola $R$, Virtanen $S M$, Hyoty $H$, Vaarala $O$, Akerblom HK. Environmental triggers and determinants of type 1 diabetes. Diabetes 2005; 54 Suppl 2:S125-36.

15. González-Sánchez JL, Serrano-Rios M. Molecular basis of insulin action. Drug News Perspect. 2007; 20(8): 527-31.

16. Hansen T. Type 2 diabetes mellitus--a multifactorial disease. Ann Univ Mariae Curie Sklodowska Med. 2002; 57(1):544-9.

17. Matthews DR, Matthews PC. Banting Memorial Lecture: reply from Matthews and Matthews. Type2 diabetes as an 'infectious'disease: is this the Black Death of the 21st century? Diabet Med. 2011; 28(7):880.

18. Wild S, Roglic G, Green A, Sicree $R$, King H. Global prevalence of diabetes: estimates for the year 2000 and projections for 2030 Diabetes Care. 2004; 27(5): 1047-53.

19. Alonso-Magdalena $P$, Vieira E, Soriano S, Menes L, Burks D, Quesada I, Nadal A. Bisphenol A exposure during pregnancy disrupts glucose homeostasis in mothers and adult male offspring. Environ Health Perspect. 2010; 118(9): 1243-50.

20. Ramachandran A, Snehalatha C, Dharmaraj D, Viswanathan M. Prevalence of glucose intolerance in Asian Indians. Urban-rural difference and significance of upper body adiposity. Diabetes Care. 1992; 15(10):1348-55.

21. Shah B. AK, Joshi P., Mahanta J., Mohan V., Thankappan $K .$, et al. Report of the surveillance of risk factors of noncommunicable diseases (STEPS 1 and 2) from five centres in India-WHO India-ICMR initiative. New Delhi, 2004.

22. Jankun J, Al-Senaidy A, Skrzypczak-Jankun E. Can inactivators of plasminogen activator inhibitor alleviate the burden of obesity and diabetes? (Review). Int J Mol Med. 2012; 29(1):3-11.

23. Centers for Disease Control and Prevention (CDC). Prevalence of diabetes and impaired fasting glucose in adultsUnited States, 1999-2000. MMWR Morb Mortal Wkly Rep. 2003; 52(35):833-7.

24. Horton, R. Diabetes - a global threat. Lancet 2009;373:1735.

25. King H, Aubert R, Herman W. Global burden of diabetes, 1995-2025: prevalence, numerical estimates, and projections. Diabetes Care. 1998; 21(9):1414-31.

26. Hogan P, Dall T, Nikolov P; American Diabetes Association. Economic costs of diabetes in the US in 2002. Diabetes Care. 2003; 26(3):917-32.

27. American Diabetes Association. Yang W, Dall TM, Halder P, Gallo P, Kowal SL, Hogan PF. Economic costs of diabetes in the U.S. in 2012.Diabetes Care 2013; 36(4):1033-46.

28. López - Jaramillo P. RJ, Goméz-Arbelaez D., Rodríguez Y., López -López I Combatir la epidemia de diabetes mellitus tipo 2 en Latino América: características especiales que demandan acciones innovadoras. J Clin Invest Arterioscl. 2011; 23(2):90-9.

29. OMS.2006.http://www.who.int/diabetes/publications/ Definition\%20and\%20diagnosis\%20of\%20diabetes_new. pdf

30. Kawate $R$. Climate and life span: regional distribution of diabetes mellitus (author's transl). Nihon Ronen Igakkai Zasshi 1980; 17(2):153-7.

31. Kim EJ KK, Lee TH, et al. The incidence of diabetes mellitus in urban and rural population in Korea. In: Baba S, Goto Y, Fukui I, eds. Diabetes Mellitus in Asia. Ecological aspects of Epidemiology, Complications and treatment. Amsterdam, The Netherlands: Excerpta Medica. 1976; 41- 44.

32. Rotimi CN, Cooper RS, Okosun IS, Olatunbosun ST, Bella $A F$, Wilks $R$, et al. Prevalence of diabetes and impaired glucose tolerance in Nigerians, Jamaicans and US blacks. Ethn Dis 1999; 9(2):190-200.

33. O'Rahilly S, Barroso I, Wareham NJ. Genetic factors in type 2 diabetes: the end of the beginning? Science. 2005; 307(5708):370-3.

34. Morgan DP, Lin LI, Saikaly HH. Morbidity and mortality in workers occupationally exposed to pesticides. Arch Environ Contam Toxicol. 1980; 9(3):349-82.

35. Kimbrough RD, Linder RE, Gaines TB. Morphological changes in livers of rats fed polychlorinated biphenyls: light microscopy and ultrastructure. Arch Environ Health. 1972; 25(5):354-64.

36. Wassermann $D$, Wassermann $M$, Lemesch $C$. Ultrastructure of beta-cells of the endocrine pancreas in rats receiving polychlorinated biphenyls. Environ Physiol Biochem. 1975; 5(5):322-40.

37. Vasiliu O, Cameron L, Gardiner J, Deguire P, Karmaus W. Polybrominated biphenyls, polychlorinated biphenyls, body weight, and incidence of adult-onset diabetes mellitus. Epidemiology. 2006; 17(4):352-9.

38. Rignell-Hydbom A, Rylander L, Hagmar L. Exposure to persistent organochlorine pollutants and type 2 diabetes mellitus. Hum Exp Toxicol. 2007; 26(5):447-52.

39. Kriska AM, Saremi A, Hanson RL, Bennett PH, Kobes S, Williams DE, et al. Physical activity, obesity, and the incidence of type 2 diabetes in a high-risk population. Am J Epidemiol. 2003; 158(7):669-75. 
40. Baker E, Blundell T, Cutfield J, Cutfield S, Dodson E, Dodson $G$, et al. The structure of $2 Z n$ pig insulin crystals at 1.5 A resolution. Philos Trans $R$ Soc Lond B Biol Sci. 1988; 319(1195):369-456.

41. Vinther T, Norrman M, Ribel U, Huus $K$, Schlein M, Steensgaard $D$, et al. Insulin analog with additional disulfide bond has increased stability and preserved activity. Protein Sci. 2013; 22(3):296-305.

42. Levine $R$, Goldstein MS. On the mechanism of action of insulin. Hormoner 1958; 11(2):2-22.

43. Park CR, Johnson LH. Effect of insulin on transport of glucose and galactose into cells of rat muscle and brain. Am J Physiol. 1955; 182(1):17-23.

44. Rowland AF, Fazakerley DJ, James DE. Mapping insulin/ GLUT4 circuitry. Traffic 2011; 12(6):672-81.

45. White $M F$, Kahn CR. The insulin signaling system. J Biol Chem. 1994; 269(1):1-4.

46. Zaid H, Antonescu CN, Randhawa VK, Klip A. Insulin action on glucose transporters through molecular switches, tracks and tethers. Biochem J. 2008; 413(2):201-15.

47. Rosen OM. After insulin binds. Science. 1987; 237(4821):1452-8.

48. O'Brien RM, Streeper R, Ayala J, Stadelmaier B, Hornbuckle L. Insulin-regulated gene expression. Biochem Soc Trans. 2001;29(Pt 4):552-8.

49. Valverde AM, Gonzalez-Rodriguez A. IRS2 and PTP1B: Two opposite modulators of hepatic insulin signalling. Arch Physiol Biochem. 2011; 117(3):105-15.

50. Belfiore A, Frasca F, Pandini G, Sciacca L, Vigneri R. Insulin receptor isoforms and insulin receptor/insulin-like growth factor receptor hybrids in physiology and disease. Endocr Rev. 2009; 30(6):586-623.

51. Gual P, Le Marchand-Brustel Y, Tanti JF. Positive and negative regulation of insulin signaling through IRS-1 phosphorylation. Biochimie. 2005; 87(1): 99-109.

52. Fritsche $L$, Weigert $C$, Haring HU, Lehmann R. How insulin receptor substrate proteins regulate the metabolic capacity of the liver--implications for health and disease. Curr Med Chem. 2008; 15(13): 1316-29.

53. Araki E, Murakami T, Shirotani T, Kanai F, Shinohara $Y$, Shimada $F$, et al. A cluster of four Sp1 binding sites required for efficient expression of the human insulin receptor gene. J Biol Chem. 1991; 266(6): 3944-8.

54. Levy JR, Krystal G, Glickman P, Dastvan F. Effects of media conditions, insulin, and dexamethasone on insulin-receptor mRNA and promoter activity in HepG2 cells. Diabetes. 1991; 40(1):58-65.

55. Webster NJ, Kong Y, Cameron KE, Resnik JL. An upstream element from the human insulin receptor gene promoter contains binding sites for C/EBP beta and NF-1. Diabetes. 1994; 43(2):305-12.

56. Yoshizato K ST, Furukawa N, Taguchi $T$, Motoshima $H$, Toyonaga T, Hirashima Y, Kawashima J, Ebina Y, Shichiri M, Araki E. Identification of a cis acting element and a novel trans-acting factor of the human insulin receptor gene in HepG2 and rat liver cells. Biochem Biophys Res Commun. 2001; 280(2):428-34.

57. Lee J, Tsai SY. Multiple hormone response elements can confer glucocorticoid regulation on the human insulin receptor gene. Mol Endocrinol. 1994; 8(5):625-34.

58. Cameron KE, ResnikJ, Webster NJ. Transcriptional regulation of the human insulin receptor promoter. J Biol Chem. 1992; 267(24):17375-83.

59. Cai D, Dhe-Paganon S, Melendez PA, Lee J, Shoelson SE.
Two new substrates in insulin signaling, IRS5/DOK4 and IRS6/DOK5. J Biol Chem. 2003; 278(28):25323-30.

60. White M. IRS proteins and the common path to diabetes. Am J Physiol Endocrinol Metab. 2002; 283(3):22.

61. White MF. Regulating insulin signaling and beta-cell function through IRS proteins. Can J Physiol Pharmacol. 2006; 84(7): 725-37.

62. Saltiel $A R$, Kahn CR. Insulin signalling and the regulation of glucose and lipid metabolism. Nature. 2001; 414(6865):799-806.

63. Alessi D. Discovery of PDK1, one of the missing links in insulin signal transduction. Colworth Medal Lecture. Biochem Soc Trans. 2001; 29(Pt 2):1-14.

64. Cantley LC. The phosphoinositide 3-kinase pathway. Science 2002; 296(5573):1655-7.

65. Gammeltoft $S$, Van Obberghen E. Protein kinase activity of the insulin receptor. Biochem J 1986; 235(1):1-11.

66. Lizcano JM, Alessi DR. The insulin signalling pathway. Curr Biol. 2002; 12(7):R236-8.

67. Hardie DG. Organismal carbohydrate and lipid homeostasis. Cold Spring Harb Perspect Biol. 2012; 1;4(5).

68. Kohn AD, Summers SA, Birnbaum MJ, Roth RA. Expression of a constitutively active Akt Ser/Thr kinase in 3T3-L1 adipocytes stimulates glucose uptake and glucose transporter 4 translocation. J Biol Chem.1996; 271(49):31372-8.

69. Kandel ES, Hay $N$. The regulation and activities of the multifunctional serine/threonine kinase Akt/PKB. Expl Cell Res. 1999; 253(1):210-29.

70. Altomare DA, Guo K, Cheng JQ, Sonoda G, Walsh K, Testa JR. Cloning, chromosomal localization and expression analysis of the mouse Akt2 oncogene. Oncogene. 1995; 11(6):1055-60.

71. Gupte AA, Bomhoff GL, Geiger PC. Age-related differences in skeletal muscle insulin signaling: the role of stress kinases and heat shock proteins. J Appl Physiol. 2008; 105(3):839-48.

72. Wu H, Bertrand KA, Choi AL, Hu FB, Laden F, Grandjean P, et al. Persistent Organic Pollutants and Type 2 Diabetes: a prospective analysis in the nurses' health study and metaanalysis. Environ Health Perspect. 2013; 121(2):153-61.

73. Cargnello M, Roux P. Activation and function of the MAPKs and their substrates, the MAPK-activated protein kinases. Microbiol Mol Biol Rev. 2011; 75(1):50-83.

74. Roy S, McPherson RA, Apolloni A, Yan J, Lane A, ClydeSmith J, et al. 14-3-3 facilitates Ras-dependent Raf-1 activation in vitro and in vivo. Mol Cell Biol. 1998; 18(7):3947-55.

75. Davis $R$. The mitogen-activated protein kinase signal transduction pathway. J Biol Chem. 1993; 268(20):14553-6.

76. Patel CJ, Bhattacharya J, Butte AJ. An Environment-Wide Association Study (EWAS) on type 2 diabetes mellitus. PloS One. 2010; 5(5):e10746.

77. Fontenele EG, Martins MR, Quidute AR, Montenegro RM $J$ r. Environmental contaminants and endocrine disruptors. Arq Bras Endocrinol Metab. 2010; 54(1):6-16.

78. Carpenter DO. Environmental contaminants as risk factors for developing diabetes. Rev Environ Health. 2008; 23(1):59-74.

79. Wang CXXS, Lv ZQ, Li YY, Wang YJ, Chen T. Exposure to persistent organic pollutants as potential risk factors for developing diabetes. Sci China Chem. 2010; 53:980- 94.

80. Milbrath MO, Wenger Y, Chang CW, Emond C, Garabrant $D$, Gillespie BW, et al. Apparent half-lives of dioxins, furans, and polychlorinated biphenyls as a function of age, body 
fat, smoking status, and breast-feeding. Environ Health Perspect. 2009; 117(3):417-25.

81. Lee DH, Lee IK, Jin SH, Steffes M, Jacobs DR, Jr. Association between serum concentrations of persistent organic pollutants and insulin resistance among nondiabetic adults: results from the National Health and Nutrition Examination Survey 1999-2002. Diabetes Care. 2007; 30(3):622-8.

82. Xu W, Wang X, Cai Z. Analytical chemistry of the persistent organic pollutants identified in the Stockholm Convention: A review. Anal Chim Acta. 2013; 6;790:1-13.

83. Codru N, Schymura MJ, Negoita S, Akwesasne Task Force on $E$, Rej $R$, Carpenter DO. Diabetes in relation to serum levels of polychlorinated biphenyls and chlorinated pesticides in adult Native Americans. Environ Health Perspect. 2007; 115(10):1442-7.

84. Rylander L, Rignell-Hydbom A, Hagmar L. A cross-sectional study of the association between persistent organochlorine pollutants and diabetes. Environ Health 2005; 4:28.

85. Hoppe AA, Carey GB. Polybrominated diphenyl ethers as endocrine disruptors of adipocyte metabolism. Obesity (Silver Spring) 2007; 15(12):2942-50.

86. Lim JS, Lee DH, Jacobs DR Jr. Association of brominated flame retardants with diabetes and metabolic syndrome in the U.S. population, 2003-2004. Diabetes Care. 2008; 31(9):1802-7.

87. Uemura H, Arisawa K, Hiyoshi M, Kitayama A, Takami $H$, Sawachika $F$, et al. Prevalence of metabolic syndrome associated with body burden levels of dioxin and related compounds among Japan's general population. Environ Health Perspect. 2009; 117(4):568-73.

88. Ruzzin J, Petersen R, Meugnier E, Madsen L, Lock EJ, Lillefosse $H$, et al. Persistent organic pollutant exposure leads to insulin resistance syndrome. Environ Health Perspect. 2010; 118(4):465-71.

89. Enan E, Matsumura F. 2,3,7,8-Tetrachlorodibenzo-p-dioxin (TCDD)-induced changes in glucose transporting activity in guinea pigs, mice, and rats in vivo and in vitro. J Biochem Toxicol. 1994; 9(2):97-106.

90. Lee DH, Steffes MW, Sjödin A, Jones RS, Needham $L L$, Jacobs DR Jr. Low dose of some persistent organic pollutants predicts type 2 diabetes: a nested case-control study. Environ Health Perspect. 2010; 118(9):1235-42.

91. Arisawa $K$, Takeda $H$, Mikasa $H$. Background exposure to PCDDs/PCDFs/PCBs and its potential health effects: a review of epidemiologic studies. J Med Invest. 2005; 52(1-2):10-21.

92. Lee $D H$, Lind $L$, Jacobs DR Jr, Salihovic $S$, van Bavel $B$, Lind PM. Associations of persistent organic pollutants with abdominal obesity in the elderly: The Prospective Investigation of the Vasculature in Uppsala Seniors (PIVUS) study. Environ Int. 2012; 40:170-8.

93. Mimura J, Fujii-Kuriyama Y. Functional role of AhR in the expression of toxic effects by TCDD. Biochim Biophys Acta. 2003; 1619(3):263-8.

94. Kouznetsova M, Huang X, Ma J, Lessner L, Carpenter DO. Increased rate of hospitalization for diabetes and residential proximity of hazardous waste sites. Environ Health Perspect. 2007; 115(1):75-9.

95. Pesatori AC, Consonni D, Bachetti S, Zocchetti C, Bonzini M, Baccarelli $A$, et al. Short- and long-term morbidity and mortality in the population exposed to dioxin after the "Seveso accident". Ind Health. 2003; 41(3):127-38.

96. Calvert GM, Sweeney MH, Deddens J, Wall DK. Evaluation of diabetes mellitus, serum glucose, and thyroid function among United States workers exposed to 2,3,7,8-tetrachlorodibenzo-p-dioxin. Occup Environ Med. 1999; 56(4):270-6

97. Henriksen GL, Ketchum NS, Michalek JE, Swaby JA. Serum dioxin and diabetes mellitus in veterans of Operation Ranch Hand. Epidemiol. 1997; 8(3):252-8.

98. Steenland K, Piacitelli L, Deddens J, Fingerhut M, Chang LI. Cancer, heart disease, and diabetes in workers exposed to 2,3,7,8-tetrachlorodibenzo-p-dioxin. J Natl Cancer Inst. 1999; 91(9):779-86.

99. Nishizumi $M$, Higaki Y. Effect of PCBs on insulin sensitivity in rats. Fukuoka Igaku Zasshi.1995; 86(5):241-6.

100. Arsenescu V, Arsenescu RI, King V, Swanson H, Cassis LA. Polychlorinated biphenyl-77 induces adipocyte differentiation and proinflammatory adipokines and promotes obesity and atherosclerosis. Environ Health Perspect. 2008; 116(6):761-8.

101. Wang SL, Tsai PC, Yang CY, Guo YL. Increased risk of diabetes and polychlorinated biphenyls and dioxins: a 24-year follow-up study of the Yucheng cohort. Diabetes Care. 2008;31(8):1574-9.

102. Turyk M, Anderson H, Knobeloch L, Imm P, Persky V. Organochlorine exposure and incidence of diabetes in a cohort of Great Lakes sport fish consumers. Environ Health Perspect. 2009;117(7):1076-82.

103. Cox S, Niskar AS, Narayan KM, Marcus M. Prevalence of self-reported diabetes and exposure to organochlorine pesticides among Mexican Americans: Hispanic health and nutrition examination survey, 1982-1984. Environ Health Perspect. 2007;115(12):1747-52.

104. CDC. Third National Report on Human Exposure to Environmental Chemicals. Atlanta, GA: Centers for Disease Control and Prevention; 2005.

105. Lassiter TL, Ryde IT, Mackillop EA, Brown KK, Levin ED, Seidler FJ, et al. Exposure of neonatal rats to parathion elicits sex-selective reprogramming of metabolism and alters the response to a high-fat diet in adulthood. Environ Health Perspect. 2008;116(11):1456-62.

106. Alonso-Magdalena $P$, Ropero $A B$, Soriano $S$, Garcia-Arevalo $M$, Ripoll C, Fuentes $E$, et al. Bisphenol-A acts as a potent estrogen via non-classical estrogen triggered pathways. Mol Cell Endocrinol. 2012; 355(2):201-7.

107. Alonso-Magdalena P, Morimoto S, Ripoll C, Fuentes $E_{\text {, }}$ Nadal A. The estrogenic effect of bisphenol A disrupts pancreatic beta-cell function in vivo and induces insulin resistance. Environ Health Perspect. 2006;114(1): 106-12.

108. Olea N, Pulgar R, Pérez P, Olea-Serrano F, Rivas A, NovilloFertrell $A$, et al. Estrogenicity of resin-based composites and sealants used in dentistry. Environ Health Perspect. 1996;104(3):298-305.

109. Munguía-López EM, Gerardo-Lugo S, Peralta E, Bolumen $S$, Soto-Valdez H. Migration of bisphenol A (BPA) from can coatings into a fatty-food simulant and tuna fish. Food Addit Contam. 2005; 22(9):892-8.

110. Vandenberg LN, Maffini MV, Sonnenschein C, Rubin BS, Soto AM. Bisphenol-A and the great divide: a review of controversies in the field of endocrine disruption. Endocr Rev. 2009; 30(1):75-95.

111. Batista TM, Alonso-Magdalena P, Vieira E, Amaral ME, Cederroth CR, Nef S, et al. Short-term treatment with bisphenol-A leads to metabolic abnormalities in adult male mice. PloS One. 2012; 7(3):e33814.

112. Ropero AB, Alonso-Magdalena P, Garcia-Garcia E, Ripoll $C$, Fuentes $E$, Nadal A. Bisphenol-A disruption of the 
endocrine pancreas and blood glucose homeostasis. Int J Androl. 2008; 31(2):194-200.

113. Ben-Jonathan N, Hugo ER, Brandebourg TD. Effects of bisphenol $A$ on adipokine release from human adipose tissue: Implications for the metabolic syndrome. Mol Cell Endocrinol. 2009; 304(1-2):49-54.

114. Lind PM, Zethelius B, Lind L. Circulating levels of phthalate metabolites are associated with prevalent diabetes in the elderly. Diabetes Care. 2012; 35(7):1519-24.

115. Rengarajan S, Parthasarathy C, Anitha M, Balasubramanian K. Diethylhexyl phthalate impairs insulin binding and glucose oxidation in Chang liver cells. Toxicology in vitro. 2007; 21(1):99-102.

116. Sweeney $M H$, Calvert GM, Egeland GA, Fingerhut MA, Halperin WE, Piacitelli LA. Review and update of the results of the NIOSH medical study of workers exposed to chemicals contaminated with 2,3,7,8-tetrachlorodibenzodioxin. Teratog Carcinog Mutagen. 1997-1998; 17(4-5):241-7.

117. Lowell BB, Shulman GI. Mitochondrial dysfunction and type 2 diabetes. Science. 2005;307(5708):384-7.

118. Kim M, Pelloux V, Guyot E, Tordjman J, Bui L-C, Chevallier $A$, et al. Inflammatory pathway genes belong to major targets of persistent organic pollutants in adipose cells. Environm Health Perspect. 2012;120(4):508-14.

119. Liu H, Biegel L, Narasimhan TR, Rowlands C, Safe S. Inhibition of insulin-like growth factor-I responses in MCF-7 cells by 2,3,7,8-tetrachlorodibenzo-p-dioxin and related compounds. Mol Cell Endocrinol. 1992;87(1-3):19-28.

120. Fujiyoshi PT, Michalek JE, Matsumura F. Molecular epidemiologic evidence for diabetogenic effects of dioxin exposure in U.S. Air force veterans of the Vietnam war. Environ Health Perspect. 2006;114(11):1677-83.

121. Liu PC, Matsumura F. Differential effects of 2,3,7,8-tetrachlorodibenzo-p-dioxin on the "adipose- type" and "brain-type" glucose transporters in mice. Mol Pharmacol. 1995;47(1):65-73.

122. Olsen L, Lind L, Lind PM. Associations between circulat- ing levels of bisphenol $A$ and phthalate metabolites and coronary risk in the elderly. Ecotoxicol Environ safety. 2012;80:179-83.

123. Kurita H, Yoshioka W, Nishimura N, Kubota N, Kadowaki T, Tohyama C. Aryl hydrocarbon receptor-mediated effects of 2,3,7,8-tetrachlorodibenzo-p-dioxin on glucosestimulated insulin secretion in mice. J Appl Toxicol: JAT. 2009;29(8):689-94.

124. Hsu H-F, Tsou T-C, Chao H-R, Kuo Y-T, Tsai F-Y, Yeh S-C. Effects of 2,3,7,8-tetrachlorodibenzo-p-dioxin on adipogenic differentiation and insulin-induced glucose uptake in 3T3-L1 cells. J Hazard Mater. 2010;182(1-3): 649-55.

125. Desvergne B, Feige J, Casals-Casas C. PPAR-mediated activity of phthalates: A link to the obesity epidemic? Mol Cell Endocrinol. 2009;304(1-2):43-8.

126. Uysal KT, Wiesbrock SM, Marino MW, Hotamisligil GS. Protection from obesity-induced insulin resistance in mice lacking TNF-alpha function. Nature. 1997; 389(6651): 610-4.

127. Kern PA, Dicker-Brown A, Said ST, Kennedy R, Fonseca $V A$. The stimulation of tumor necrosis factor and inhibition of glucose transport and lipoprotein lipase in adipose cells by 2,3,7,8-tetrachlorodibenzo-p-dioxin. Metabolism. 2002;51(1):65-8.

128. Hokanson R, Miller S, Hennessey M, Flesher M, Hanneman $W$, Busbee D. Disruption of estrogen-regulated gene expression by dioxin: downregulation of a gene associated with the onset of non-insulin-dependent diabetes mellitus (type 2 diabetes). Hum Exp Toxicol. 2004;2 3(12): 555-64.

129. Savage DB, Petersen KF, Shulman GI. Disordered lipid metabolism and the pathogenesis of insulin resistance. Physiol Rev. 2007;87(2):507-20.

130. Baroni MG, Oelbaum RS, Pozzilli P, Stocks J, Li SR, Fiore $V$, et al. Polymorphisms at the GLUT1 (HepG2) and GLUT4 (muscle/adipocyte) glucose transporter genes and noninsulin-dependent diabetes mellitus (NIDDM). Hum Genet. 1992;88(5):557-61. 